\title{
A FAST NUMERICAL ALGORITHM FOR THE INTEGRATION OF RATIONAL FUNCTIONS
}

\author{
DANTE MANNA, LUIS MEDINA, VICTOR H. MOLL, AND ARMIN STRAUB
}

\begin{abstract}
A new iterative method for high-precision numerical integration of rational functions on the real line is presented. The algorithm transforms the rational integrand into a new rational function preserving the integral on the line. The coefficients of the new function are explicit polynomials in the original ones. These transformations depend on the degree of the input and the desired order of the method. Both parameters are arbitrary. The formulas can be precomputed. Iteration yields an approximation of the desired integral with $m$-th order convergence. Examples illustrating the automatic generation of these formulas and the numerical behaviour of this method are given.
\end{abstract}

\section{INTRODUCTION}

The numerical integration of the elliptic integral

$$
G(a, b)=\int_{0}^{\pi / 2} \frac{d \theta}{\sqrt{a^{2} \cos ^{2} \theta+b^{2} \sin ^{2} \theta}}
$$

can be accomplished by iterating the transformation

$$
\mathcal{L}_{e}: \mathbb{R}^{2} \rightarrow \mathbb{R}^{2}, \quad(a, b) \mapsto\left(\frac{a+b}{2}, \sqrt{a b}\right) .
$$

Gauss [6] established that $G(a, b)$ is invariant under the transformation $\mathcal{L}_{e}$, i.e.,

$$
G\left(\mathcal{L}_{e}(a, b)\right)=G(a, b) .
$$

Moreover, the iterates $\left(a_{n}, b_{n}\right)$ defined recursively by $\left(a_{0}, b_{0}\right)=(a, b)$ and $\left(a_{n}, b_{n}\right)=$ $\mathcal{L}_{e}\left(a_{n-1}, b_{n-1}\right)$ for $n \geq 1$, satisfy

$$
\left|a_{n+1}-b_{n+1}\right| \leq \frac{1}{2}\left|a_{n}-b_{n}\right|^{2},
$$

illustrating the quadratic convergence of $a_{n}$ and $b_{n}$ to a common limit $\operatorname{AGM}(a, b)$. This is the arithmetic-geometric mean of $a$ and $b . \mathcal{L}_{e}$ is known as the elliptic Landen transformation. The reader will find in [8] a survey of the diverse aspects of this transformation and its generalizations.

The invariance of the integral $G(a, b)$ under $\mathcal{L}_{e}$ yields

$$
G(a, b)=\frac{\pi}{2} \operatorname{AGM}^{-1}(a, b) .
$$

In particular, the value of the elliptic integral $G(a, b)$ can be approximated using the iterates $a_{n}$ (or $b_{n}$ ). The functions $G(a, b)$ and $\operatorname{AGM}(a, b)$ along with the formula

Date: August 13, 2010.

1991 Mathematics Subject Classification. Primary 65D30, Secondary 33F05.

Key words and phrases. Numerical integration, Landen transformations. 
(1.5), are at the center of highly effective computation of the basic constants of Analysis [2].

A scheme for the computation of the rational integral

$$
F(a, b)=\int_{-\infty}^{\infty} \frac{b_{0} x^{p-2}+\ldots+b_{p-3} x+b_{p-2}}{a_{0} x^{p}+\ldots+a_{p-1} x+a_{p}} d x
$$

with $a=\left(a_{0}, a_{1}, \ldots, a_{p}\right), b=\left(b_{0}, b_{1}, \ldots, b_{p-2}\right)$, is presented here. Rational Landen transformations

$$
\mathcal{L}_{m, p}: \mathbb{C}^{2 p} \rightarrow \mathbb{C}^{2 p}, \quad(a, b) \mapsto \mathcal{L}_{m, p}(a, b)
$$

which preserve the integral $F(a, b)$ are constructed. Iteration of $\mathcal{L}_{m, p}$ yields a sequence of coefficients $\left(a_{n, 0}, a_{n, 1}, \cdots, a_{n, p}\right)$ and $\left(b_{n, 0}, b_{n, 1}, \cdots, b_{n, p-2}\right)$ for a sequence of rational functions which are shown to converge to a constant multiple of $1 /\left(x^{2}+1\right)$. The invariance of the integral $F(a, b)$ under $\mathcal{L}_{m, p}$ yields

$$
F(a, b)=c \int_{-\infty}^{\infty} \frac{d x}{x^{2}+1}=\pi c=\pi \lim _{n \rightarrow \infty} \frac{b_{n, 0}}{a_{n, 0}},
$$

that determines the constant $c$. The sequence $\left\{\pi b_{n, 0} / a_{n, 0}: n=1,2,3 \ldots\right\}$ of approximations to the integral $F(a, b)$ converges with order $m$; that is, the error $e_{n}:=\left|\pi b_{n, 0} / a_{n, 0}-F(a, b)\right|$ satisfies

$$
\left|e_{n+1}-e_{n}\right| \leq C\left|e_{n}-e_{n-1}\right|^{m}
$$

with an absolute constant $C$.

The outlined algorithm for computing rational integrals over the real line, presented in more detail in Section 6, consists of the two parts:

- Creation of the rational Landen transformation $\mathcal{L}_{m, p}$ where $m$ is the desired order of convergence and $p$ is the degree of the denominator of the rational function to be integrated. This is discussed in Section 2.

- Iteration of the Landen transformation, which is analyzed in Sections 3 and 6 with the view towards complexity and implementation, respectively. Numerical examples of this method are discussed in Section 4.

Remark 1.1. Given $m$ and $p$, the map $\mathcal{L}_{m, p}$ can be precomputed and the result can be stored for use in the second part of the algorithm. Therefore, the first part of the algorithm carries a one-time cost and is not figured into the complexity of the method which is discussed in Section 3.

Remark 1.2. The numerical method for the integration of rational functions presented here is different in spirit than the standard ones: the approximation to the integral is obtained from a recurrence acting on the coefficients of the integrand. In particular, the domain of integration is not discretized and the integrand is never evaluated. Examples that illustrate the method and a study of the cost involved are presented.

Future work. The algorithm presented here is restricted to integrals on the whole line. The extension to a finite interval requires the development of Landen transformations on the half line. This question is open, even for the simplest case of

$$
I_{2}(a, b, c):=\int_{0}^{\infty} \frac{d x}{a x^{2}+b x+c} .
$$


The method has been coupled with Pade approximations [1] to produce a fast, robust integration algorithm for some non-rational integrands; namely, those of the form $R(\mu(x)) \mu^{\prime}(x)$ for a rational function $R$ and an increasing change of scale $\mu$. Extensions to all smooth integrands remain to be completed.

\section{The LANDEN TRANSFORMATION}

In this section we present simple examples, and then describe the algorithm in detail. The first two examples show the transformations $\mathcal{L}_{2,2}$ and $\mathcal{L}_{3,4}$. Both maps are given by polynomial functions.

Example 2.1. The rational Landen transformation $\mathcal{L}_{2,2}$ is given by

$$
\mathcal{L}_{2,2}\left(b_{0}, a_{0}, a_{1}, a_{2}\right)=\left(b_{0}^{\prime}, a_{0}^{\prime}, a_{1}^{\prime}, a_{2}^{\prime}\right),
$$

with

$$
\begin{aligned}
b_{0}^{\prime} & =2 a_{0} b_{0}+2 a_{2} b_{0}, \\
a_{0}^{\prime} & =4 a_{0} a_{2}, \\
a_{1}^{\prime} & =-2 a_{0} a_{1}+2 a_{1} a_{2}, \\
a_{2}^{\prime} & =a_{0}^{2}-a_{1}^{2}+2 a_{0} a_{2}+a_{2}^{2} .
\end{aligned}
$$

Example 2.2. The rational Landen transformation $\mathcal{L}_{3,4}$ is computed as

$$
\mathcal{L}_{3,4}\left(b_{0}, b_{1}, b_{2}, a_{0}, a_{1}, a_{2}, a_{3}, a_{4}\right)=\left(b_{0}^{\prime}, b_{1}^{\prime}, b_{2}^{\prime}, a_{0}^{\prime}, a_{1}^{\prime}, a_{2}^{\prime}, a_{3}^{\prime}, a_{4}^{\prime}\right)
$$

with

$$
\begin{aligned}
& b_{0}^{\prime}=3 a_{0}^{2} b_{0}-a_{1}^{2} b_{0}+10 a_{0} a_{2} b_{0}+3 a_{2}^{2} b_{0}-6 a_{1} a_{3} b_{0}-9 a_{3}^{2} b_{0}+\ldots \\
& a_{0}^{\prime}=a_{0}^{3}-3 a_{0} a_{1}^{2}+6 a_{0}^{2} a_{2}+9 a_{0} a_{2}^{2}-18 a_{0} a_{1} a_{3}-27 a_{0} a_{3}^{3}+18 a_{0}^{2} a_{4}+\ldots
\end{aligned}
$$

and so on. The point to be made here is that, while the formulas for the transformations $\mathcal{L}_{m, p}$ grow in size as $m$ and $p$ increase, these formulas only have to be computed once.

Remark 2.3. The rational Landen transformations $\mathcal{L}_{m, p}$ are given by polynomial equations which are homogeneous of degree $m$. Furthermore, the $a_{i}^{\prime}$ depend only on the $a_{i}$.

Remark 2.4. The invariance of $F(a, b)$ under $\mathcal{L}_{m, p}$ implies that the set

$$
\mathcal{R}=\left\{(a, b) \in \mathbb{R}^{2 p}: \quad F(a, b) \text { is finite }\right\}
$$

is preserved by $\mathcal{L}_{m, p}$. The action of $\mathcal{L}_{m, p}$ outside of $\mathcal{R}$ is difficult to analyze. The reader will find in [9] some illustrations for $\mathcal{L}_{2,6}$.

Remark 2.5. The dynamical study of $\mathcal{L}_{2,6}$ appeared in [4]. An extension of this work to $\mathcal{L}_{3,6}$ and $\mathcal{L}_{4,6}$ will appear in [3] and [5], respectively.

Example 2.6. Iterating $\mathcal{L}_{2,2}$ with initial conditions $a_{0, i}=a_{i}, b_{0, i}=b_{i}$ yields a sequence $\left(b_{n, 0}, a_{n, 0}, a_{n, 1}, a_{n, 2}\right)$, defined by

$$
\left(b_{n+1,0}, a_{n+1,0}, a_{n+1,1}, a_{n+1,2}\right):=\mathcal{L}_{2,2}\left(b_{n, 0}, a_{n, 0}, a_{n, 1}, a_{n, 2}\right),
$$

which satisfies

$$
\int_{-\infty}^{\infty} \frac{b_{n, 0} d x}{a_{n, 0} x^{2}+a_{n, 1} x+a_{n, 2}}=\int_{-\infty}^{\infty} \frac{b_{0} d x}{a_{0} x^{2}+a_{1} x+a_{2}}
$$


The convergence result in Section 5 shows that

$$
\frac{b_{n, 0}}{a_{n, 0} x^{2}+a_{n, 1} x+a_{n, 2}} \sim \frac{b_{n, 0}}{a_{n, 0}} \frac{1}{x^{2}+1}
$$

as $n \rightarrow \infty$. Furthermore the convergence is quadratic. Therefore,

$$
\int_{-\infty}^{\infty} \frac{b_{0} d x}{a_{0} x^{2}+a_{1} x+a_{2}}=\pi \lim _{n \rightarrow \infty} \frac{b_{n, 0}}{a_{n, 0}} .
$$

For instance, starting with $1 /\left(x^{2}+4 x+15\right)$, the algorithm produces $\mathcal{L}_{2,2}(1,1,4,15)=$ $(32,60,112,240)$. Hence,

$$
\int_{-\infty}^{\infty} \frac{d x}{x^{2}+4 x+15}=\int_{-\infty}^{\infty} \frac{32 d x}{60 x^{2}+112 x+240} .
$$

The first two terms of the approximating sequence $I_{n}=\pi a_{n, 0} / b_{n, 0}$ are given by $I_{0}=\pi$ and $I_{1}=32 \pi / 60$. These approximations converge to $\pi / \sqrt{11}$, the exact value of the integral. The error $e_{n}:=\left|I_{n}-\pi / \sqrt{11}\right|$ and the relative approximate error $d_{n}:=\left|\left(I_{n+1}-I_{n}\right) / I_{n+1}\right|$ are given in Table 1 . Observe that 19 iterations provide about 100,000 correct digits. Furthermore, this precision can be reached by using just slightly more, say 5 , than 100, 000 digits of working precision.

\begin{tabular}{||c||c|c|c|c|c|c|c||}
\hline$n$ & 1 & 2 & 3 & 4 & 5 & 10 & 20 \\
\hline$e_{n}$ & 0.73 & 0.10 & 0.034 & 0.00042 & $1.2 \cdot 10^{-6}$ & $7.8 \cdot 10^{-197}$ & $7.0 \cdot 10^{-200,886}$ \\
\hline$d_{n}$ & 0.60 & 0.15 & 0.036 & 0.00044 & $1.3 \cdot 10^{-6}$ & $8.2 \cdot 10^{-197}$ & $7.4 \cdot 10^{-200,886}$ \\
\hline
\end{tabular}

TABLE 1. Absolute and relative approximate errors for a method of order 2 .

The creation of the rational Landen transformation formulas depends on two polynomial sequences. Let $m \geq 2$ be an integer. Define the polynomials

$$
\begin{aligned}
P_{m}(x) & =\sum_{j=0}^{\lfloor m / 2\rfloor}(-1)^{j}\left(\begin{array}{c}
m \\
2 j
\end{array}\right) x^{m-2 j} \\
Q_{m}(x) & =\sum_{j=0}^{\lfloor(m-1) / 2\rfloor}(-1)^{j}\left(\begin{array}{c}
m \\
2 j+1
\end{array}\right) x^{m-(2 j+1)},
\end{aligned}
$$

that come from the relation

$$
\cot (m \theta)=R_{m}(\cot \theta)
$$

satisfied by $R_{m}=P_{m} / Q_{m}$. Details about these polynomials can be found in [7].

Let $A, B$ be polynomials. The change of variables $y=R_{m}(x)$ yields a new pair of polynomials $A_{1}, B_{1}$ such that

$$
\int_{-\infty}^{\infty} \frac{B_{1}(x)}{A_{1}(x)} d x=\int_{-\infty}^{\infty} \frac{B(x)}{A(x)} d x
$$

The change of variables requires the domain to be splitted according to the branches of the inverse $R_{m}^{-1}$. These are specified by the intervals $\left(q_{j-1}, q_{j}\right)$ where $q_{0}=-\infty$, $q_{j}=\cot (\pi j / m)$ for $1 \leq j \leq m-1$, and $q_{m}=+\infty$. The function $y=R_{m}(x)$ is 
invertible on each of the subintervals $\left(q_{j-1}, q_{j}\right)$, and the (local) inverse is denoted by $x=\omega_{j}(y)$. After substituting $y=R_{m}(x)$ in each interval, it follows that

$$
\int_{-\infty}^{\infty} \frac{B(x)}{A(x)} d x=\sum_{j=1}^{m} \int_{q_{j-1}}^{q_{j}} \frac{B(x)}{A(x)} d x=\int_{-\infty}^{\infty} \sum_{j=1}^{m} \frac{B\left(\omega_{j}(y)\right)}{A\left(\omega_{j}(y)\right)} \omega_{j}^{\prime}(y) d y .
$$

The integrand on the right-hand side of (2.5) is indeed a rational function $B_{1} / A_{1}$; see [7]. The rational Landen transformation $\mathcal{L}_{m}: \mathbb{C}(x) \rightarrow \mathbb{C}(x)$ is defined by $B / A \mapsto B_{1} / A_{1}$. The full details are given below.

Step 1: The rational function $R_{m}=P_{m} / Q_{m}$ comes from (2.2). First construct the polynomial

$$
A_{1}(x):=\operatorname{Res}\left(A, P_{m}-x Q_{m}\right)
$$

where Res denotes the resultant. The degrees of the polynomials involved are $p:=\operatorname{deg} A$ and $m=\operatorname{deg}\left(P_{m}-x Q_{m}\right)$, respectively. The degree of the denominator is preserved; that is, $\operatorname{deg}\left(A_{1}\right)=\operatorname{deg}(A)$. The coefficients of $A_{1}$ are polynomials in those of $A$.

Step 2: The polynomial $E_{m}(x):=\left[P_{m}(x)\right]^{p} A_{1}\left(R_{m}(x)\right)$ is a multiple of $A$. Compute the quotient

$$
C(x):=E(x) \frac{B(x)}{A(x)}=\sum_{k=0}^{s} c_{k} x^{s-k}
$$

with $s:=m p-2$.

Step 3: Define the expressions

$$
\begin{aligned}
& T_{x}(a, b):= \sum_{j=0}^{x}(-1)^{a-x+j}\left(\begin{array}{c}
a \\
x-j
\end{array}\right)\left(\begin{array}{l}
b \\
j
\end{array}\right), \\
& M_{1}(j, \alpha, \beta, \gamma):=\quad(-1)^{j+\alpha-\beta} c_{2 j} \frac{2^{2(\alpha-\beta)} \alpha}{2 \alpha-\beta}\left(\begin{array}{c}
2 \alpha-\beta \\
\beta
\end{array}\right)\left(\begin{array}{c}
\nu-\alpha-1+\beta \\
\gamma
\end{array}\right) \times \\
&\left(T_{\lambda+\alpha m}(2 j, s-2 j)+T_{\lambda-\alpha m}(2 j, s-2 j)\right), \\
& M_{2}(j, \alpha, \beta, \gamma):=(-1)^{j+\beta} c_{2 j+1} 2^{2 \beta+1}\left(\begin{array}{c}
\alpha+\beta \\
2 \beta+1
\end{array}\right)\left(\begin{array}{c}
\nu-2-\beta \\
\gamma
\end{array}\right) \times \\
&\left(T_{\lambda+\alpha m}(2 j+1, s-2 j-1)-T_{\lambda-\alpha m}(2 j+1, s-2 j-1)\right),
\end{aligned}
$$

where $\nu:=p / 2$ and $\lambda:=(m p-2) / 2$. 
Step 4: Define

$$
\begin{aligned}
B_{1}(x) & :=\frac{1}{2^{s}} \sum_{\gamma=0}^{\nu-1}\left(\left(\begin{array}{c}
\nu-1 \\
\gamma
\end{array}\right) \sum_{j=0}^{\lambda}(-1)^{j} c_{2 j} T_{\lambda}(2 j, s-2 j)\right) x^{2 \gamma} \\
& +\frac{1}{2^{s}} \sum_{\gamma=0}^{\nu-2}\left(\sum_{j=0}^{\lambda} \sum_{\alpha=1}^{\nu-1-\gamma} \sum_{\beta=0}^{\alpha} M_{1}(j, \alpha, \beta, \gamma)\right) x^{2 \gamma} \\
& +\frac{1}{2^{s}} \sum_{\gamma=1}^{\nu-1}\left(\sum_{j=0}^{\lambda} \sum_{\alpha=\nu-\gamma}^{\nu-1} \sum_{\beta=\alpha-\nu+\gamma+1}^{\alpha} M_{1}(j, \alpha, \beta, \gamma)\right) x^{2 \gamma} \\
& +\frac{1}{2^{s}} \sum_{\gamma=0}^{\nu-2}\left(\sum_{j=0}^{\lambda-1} \sum_{\alpha=1}^{\nu-1-\gamma} \sum_{\beta=0}^{\alpha-1} M_{2}(j, \alpha, \beta, \gamma)\right) x^{2 \gamma+1} \\
& +\frac{1}{2^{s}} \sum_{\gamma=1}^{\nu-2}\left(\sum_{j=0}^{\lambda-1} \sum_{\alpha=\nu-\gamma}^{\nu-1} \sum_{\beta=0}^{\alpha-1} M_{2}(j, \alpha, \beta, \gamma)\right) x^{2 \gamma+1} .
\end{aligned}
$$

The following theorem has been established in [7].

Theorem 2.7. The rational function $B_{1} / A_{1}$ satisfies

$$
\int_{-\infty}^{\infty} \frac{B_{1}(x)}{A_{1}(x)} d x=\int_{-\infty}^{\infty} \frac{B(x)}{A(x)} d x
$$

whenever one of the integrals is finite. Moreover $\operatorname{deg} A_{1}=\operatorname{deg} A$.

Definition 2.1. The rational Landen transformation $\mathcal{L}_{m}: \mathbb{C}(x) \rightarrow \mathbb{C}(x)$ is defined by $\mathcal{L}_{m}(B / A)=B_{1} / A_{1}$. When $\mathcal{L}_{m}$ is acting on the coefficients of a rational function of degree $p$ we write $\mathcal{L}_{m, p}\left(b_{0}, b_{1}, \ldots, b_{p-2}, a_{0}, a_{1}, \ldots, a_{p}\right)$ as in (1.7) and Example 2.6 .

Precomputed formulas for the $\mathcal{L}_{m, p}$ as well as a program written in Mathematica that generates these formulas following the above algorithm and featuring the numerical integration of rational functions over the real line, as outlined in the introduction, are available for download from the authors. Some details of the implementation will be discussed in Section 6 .

\section{Complexity of the Algorithm}

This section discusses the complexity of computing definite integrals using Landen transformations. The analysis is restricted to the cost of one iteration. The actual generation of the Landen transformation is not considered since it is a onetime cost.

Examples 2.1 and 2.2 illustrate the fact that $\mathcal{L}_{m, p}$ is a mapping $\mathbb{C}^{2 p} \rightarrow \mathbb{C}^{2 p}$ defined by polynomial equations with integer coefficients. Assume that these polynomial equations appear in expanded form. The number of multiplications $c_{m, p}$ involved in computing $\mathcal{L}_{m, p}$ not including multiplications with constants is now counted. Additions and multiplying with constants have lower complexity than multiplication which is why they are not included in this count. 
Example 3.1. Example 2.1 gives $\mathcal{L}_{2,2}\left(b_{0}, a_{0}, a_{1}, a_{2}\right)=\left(b_{0}^{\prime}, a_{0}^{\prime}, a_{1}^{\prime}, a_{2}^{\prime}\right)$ with

$$
\begin{aligned}
b_{0}^{\prime} & =2 a_{0} b_{0}+2 a_{2} b_{0}, \\
a_{0}^{\prime} & =4 a_{0} a_{2}, \\
a_{1}^{\prime} & =-2 a_{0} a_{1}+2 a_{1} a_{2}, \\
a_{2}^{\prime} & =a_{0}^{2}-a_{1}^{2}+2 a_{0} a_{2}+a_{2}^{2} .
\end{aligned}
$$

Hence $\mathcal{L}_{2,2}$ requires $c_{2,2}=9$ multiplications. More values $c_{m, p}$ are given in Table 2 .

\begin{tabular}{||c||c|c|c|c||}
\hline$p \backslash m$ & 2 & 3 & 4 & 5 \\
\hline 2 & 9 & 32 & 75 & 144 \\
4 & 36 & 204 & 702 & 1896 \\
6 & 94 & 756 & 3492 & 12040 \\
8 & 195 & 2056 & 11895 & 49712 \\
10 & 351 & 4600 & 31923 & 156512 \\
12 & 574 & 9012 & 72858 & 409688 \\
\hline
\end{tabular}

TABLE 2. Number of operations involved in $\mathcal{L}_{m, p}$.

The data above suggest that $c_{m, p}=O\left(p^{m+1}\right)$. Moreover, for $m=2$ and $m=3$ the number of multiplications $c_{m, 2 p}$ seem to be exactly

$$
\begin{aligned}
c_{2,2 p} & =\frac{1}{2}(p+1)\left(2+3 p+4 p^{2}\right) \\
c_{3,2 p} & =\frac{2}{3} p\left(15+13 p+12 p^{2}+8 p^{3}\right) .
\end{aligned}
$$

Remark 3.2. As noted in Remark 2.3, the Landen transformation $\mathcal{L}_{m, p}$ only involves monomials of degree $m$. Therefore, $c_{m, p}$ is the number of these monomials times $m-1$. Writing the polynomial expressions defining $\mathcal{L}_{m, p}$ in a different form, one may hope to decrease the cost of its computation. Experiments conducted in Mathematica show that writing these polynomials in multivariate Horner form decreases the order of growth to $O\left(p^{m}\right)$.

Remark 3.3. From a practical point of view, the Landen transformations of order 2 are generally preferable to higher order ones. This is because combining $n$ Landen iterations $\mathcal{L}_{2, p}$ into one step gives a method of order $2^{n}$ (in fact, $\mathcal{L}_{2, p}^{n}=\mathcal{L}_{2^{n}, p}$ ) which requires $n c_{2, p}$ multiplications. Multiple experiments show that $n c_{2, p} \ll c_{2^{n}, p}$.

\section{Some numerical EXAmples}

In this section two examples that illustrate the procedure described in this work are presented.

Example 4.1. This first example illustrates the behavior on highly oscillatory integrands which, as it turns out, is basically no different from the behaviour on more regular integrands. A Landen transformation of order 2 is applied to the rational function

$$
f_{k}(x)=\frac{2^{k} P_{k}(x / 2)}{\left(\begin{array}{c}
k \\
k k / 2\rfloor
\end{array}\right)\left(x^{2 k}+1\right)},
$$




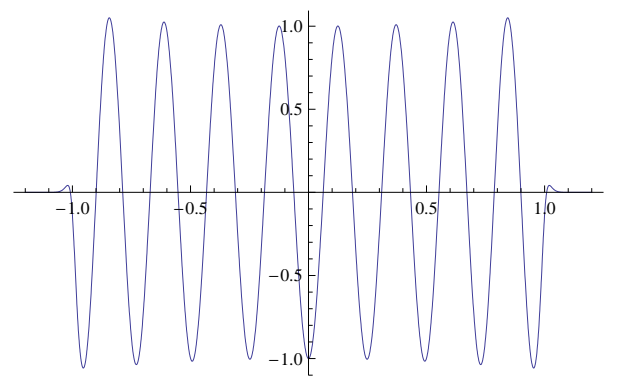

Figure 1. The oscillatory rational function $f_{50}(x)$

where $P_{k}$ is the Legendre polynomial. The normalization factor is chosen so that $\left|f_{k}(0)\right|=1$ for even $k$.

The number of steps $n_{k}(d)$ needed for the relative error to drop below $10^{-d}$ is tabulated in Table 3 . These calculations only require a working precision of a few more, say 5 , than $d$ digits. Observe that, as expected, to obtain the 10 fold precision only about $\log (2,10) \approx 3.32$ additional iterations are necessary.

\begin{tabular}{||c||c|c|c|c|c|c|c|c|c||}
\hline$k$ & 2 & 4 & 6 & 8 & 10 & 20 & 30 & 40 & 50 \\
\hline$n_{k}(20)$ & 6 & 7 & 8 & 8 & 9 & 10 & 10 & 11 & 11 \\
$n_{k}(50)$ & 8 & 9 & 9 & 10 & 10 & 11 & 12 & 12 & 12 \\
$n_{k}(100)$ & 9 & 10 & 10 & 11 & 11 & 12 & 13 & 13 & 13 \\
$n_{k}(1000)$ & 12 & 13 & 14 & 14 & 14 & 15 & 16 & 16 & 17 \\
$n_{k}(10000)$ & 15 & 16 & 17 & 17 & 18 & 19 & 19 & 20 & 20 \\
\hline
\end{tabular}

TABLE 3. Number of steps $n_{k}(d)$ needed to get relative error less than $10^{-d}$.

Example 4.2. The method proposed here is also applicable to problems that are nearly singular, that is, to rational functions with poles arbitrarily close to the real axis. For fixed $\epsilon>0$, apply $\mathcal{L}_{2,2}$ to the rational function

$$
f_{\epsilon}(x)=\frac{1}{(x-1)^{2}+\epsilon^{2}}=\frac{1}{x^{2}-2 x+\left(1+\epsilon^{2}\right)}
$$

which over the real line integrates to $1 / \epsilon$. For decreasing values of $\epsilon=10^{-k}$ the number of steps $n_{k}(d)$ needed so that the relative error is less than $10^{-d}$ can be found in Table 4. In this case, it is sufficient to use a working precision of $d+2 k$. The data in Table 4 suggest that the number of steps $n_{k}(d)$ grows linear in $k$ and, as expected, logarithmic in $d$.

\section{Convergence of Landen iterates}

In this section the convergence of the iterates of the Landen transformation $\mathcal{L}_{m, p}$ starting at the rational function

$$
f(x)=\frac{B(x)}{A(x)}=\frac{b_{0} x^{p-2}+\ldots+b_{p-3} x+b_{p-2}}{a_{0} x^{p}+\ldots+a_{p-1} x+a_{p}}
$$




\begin{tabular}{||c||c|c|c|c|c|c|c|c||}
\hline$k$ & 1 & 2 & 3 & 4 & 5 & 10 & 20 & 40 \\
\hline$n_{k}(20)$ & 9 & 13 & 16 & 19 & 23 & 39 & 72 & 139 \\
$n_{k}(50)$ & 11 & 14 & 17 & 21 & 24 & 41 & 74 & 140 \\
$n_{k}(100)$ & 12 & 15 & 18 & 22 & 25 & 42 & 75 & 141 \\
$n_{k}(1000)$ & 15 & 18 & 22 & 25 & 28 & 45 & 78 & 145 \\
$n_{k}(10000)$ & 18 & 22 & 25 & 28 & 32 & 48 & 81 & 148 \\
\hline
\end{tabular}

TABLE 4. Number of steps $n_{k}(d)$ needed to get relative error less than $10^{-d}$ when integrating $f_{10^{-k}}$.

is considered. Denote by $f_{n}=B_{n} / A_{n}$ the Landen iterates $\mathcal{L}_{m, p}^{n}(f)$. Assuming that $f$ has no poles on the real line, Theorem 5.4 shows that, as $n \rightarrow \infty$,

$$
f_{n} \rightarrow \frac{c}{x^{2}+1}
$$

where $c$ is determined by the integral of $f$ (and vice versa). Moreover, convergence is of order $m$. This implies the convergence of the coefficients of $A_{n}=a_{n, 0} x^{p}+$ $a_{n, 1} x^{p-1}+\ldots+a_{n, p}$ and $B_{n}=b_{n, 0} x^{p-2}+b_{n, 1} x^{p-3}+\ldots+b_{n, p-2}$ as described in the following proposition.

Proposition 5.1. Let $\lambda_{+}$be the number of roots of $A$ with positive imaginary part and $\lambda_{-}$the number of roots with negative imaginary part (note that $\lambda_{+}+\lambda_{-}$ is the degree of $A$ ). Then for the constant $c$ defined in (5.1)

$$
\frac{B_{n}}{a_{n, 0}} \rightarrow c(x-i)^{\lambda_{+}-1}(x+i)^{\lambda_{-}-1}, \quad \frac{A_{n}}{a_{n, 0}} \rightarrow(x-i)^{\lambda_{+}}(x+i)^{\lambda_{-}} .
$$

Note that the constant $c$ in (5.1) vanishes if either $\lambda_{+}=0$ or $\lambda_{-}=0$.

Proof. The proof is given for $m=2$, the general case can be established by similar methods. Recall that the denominator $A_{n}$ only depends on $A$ and it is transformed according to

$$
A_{n+1}(x)=\operatorname{Res}_{z}\left(A_{n}(z), z^{2}-2 x z-1\right) .
$$

Hence, if $A_{n} / a_{n, 0}=\prod_{k}\left(x-\lambda_{n, k}\right)$ then

$$
A_{n+1} / a_{n, 0}=\prod_{k}\left(\lambda_{n, k}^{2}-2 \lambda_{n, k} x-1\right) .
$$

Therefore the roots of $A_{n+1}(x)$ are $\lambda_{n+1, k}=\frac{\lambda_{n, k}^{2}-1}{2 \lambda_{n, k}}$. Note that

$$
\operatorname{Im}\left(\frac{\lambda^{2}-1}{2 \lambda}\right)=\frac{1}{2}\left(1+\frac{1}{|\lambda|^{2}}\right) \operatorname{Im}(\lambda)
$$

which implies that the signs of the imaginary part of the roots of $A$ are preserved by the Landen iterations. In particular, this shows that the integrablity of a rational function is preserved by $\mathcal{L}_{m, p}$.

The transformation $\lambda \mapsto \frac{\lambda^{2}-1}{2 \lambda}=\lambda-\frac{\lambda^{2}+1}{2 \lambda}$ is the Newton map of $\lambda^{2}+1$. Therefore, each root $\lambda_{n, k}$ converges to $i$ or $-i$ depending on the sign of $\operatorname{Im} \lambda_{1, k}$. Furthermore, the Newton map is known to exhibit quadratic convergence. This establishes the result about $A_{n}$. Theorem 5.4 shows that $B_{n} / A_{n} \rightarrow c /\left(x^{2}+1\right)$. This gives the corresponding result for $B_{n}$. 
Corollary 5.2. If $A$ has only real coefficients, then $p$ is even and

$$
\frac{B_{n}}{a_{n, 0}} \rightarrow c\left(x^{2}+1\right)^{p / 2-1}, \quad \frac{A_{n}}{a_{n, 0}} \rightarrow\left(x^{2}+1\right)^{p / 2} .
$$

Remark 5.1. An explicit and curious formula for the denominator $A_{1}$ of $\mathcal{L}_{2}(1 / A)$ is described next. This is an expression independent of the roots of $A$. In the computation of $A_{1}(x)=\operatorname{Res}_{z}\left(A(z), z^{2}-2 x z-1\right)$, reduce $A(z)$ modulo $z^{2}-2 x z-1$ before computing the resultant. Proceeding in a recursive manner, write $z^{n} \equiv$ $a_{n}(x)+b_{n}(x) z$. Then $z^{2} \equiv 1+2 x z$ leads to the recurrence

$$
a_{n+1}(x)=b_{n}(x), \quad b_{n+1}(x)=a_{n}(x)+2 x b_{n}(x) .
$$

Therefore $z^{n} \equiv b_{n-1}(x)+b_{n}(x) z$ where $b_{n}(x)$ is defined by

$$
b_{n+1}(x)=2 x b_{n}(x)+b_{n-1}(x), \quad b_{0}=0, b_{1}=1 .
$$

It follows that $z^{n} \equiv F_{n-1}(2 x)+F_{n}(2 x) z$ where $F_{n}(x)$ is the $n$th Fibonacci polynomial. These polynomials are defined recursively by $F_{0}(x)=0, F_{1}(x)=1$ and $F_{n+1}(x)=x F_{n}(x)+F_{n-1}(x)$ and they are explicitely given by

$$
F_{n+1}(x)=\sum_{k=0}^{\lfloor n / 2\rfloor}\left(\begin{array}{c}
n-k \\
k
\end{array}\right) x^{n-2 k} .
$$

If $A(z)=\sum a_{k} z^{k}$ then $A(z) \equiv a(x)+b(x) z$ for $a(x)=\sum a_{k} F_{k-1}(2 x)$ and $b(x)=\sum a_{k} F_{k}(2 x)$. It follows that

$$
\begin{aligned}
A_{1}(x) & =a(x)^{2}-b(x)^{2}+2 a(x) b(x) x \\
& =a(x)(a(x)+2 x b(x))-b(x)^{2} \\
& =\left(\sum a_{k} F_{k-1}\right)\left(\sum a_{k} F_{k+1}\right)-\left(\sum a_{k} F_{k}\right)^{2}
\end{aligned}
$$

where $F_{k}$ is used to abbreviate $F_{k}(2 x)$.

Remark 5.2. The proof of Proposition 5.1 contains explicitly the transformation of the denominator under the Landen transformation $\mathcal{L}_{2}$ in terms of its roots. In particular,

$$
\mathcal{L}_{2}\left(\frac{1}{x-\lambda}\right)=\frac{-2 \lambda}{-2 \lambda x+\lambda^{2}-1}=\frac{1}{x-\frac{\lambda^{2}-1}{2 \lambda}} .
$$

For a rational function $f=B / A$, with no repeated poles off the real line and $\operatorname{deg}(B) \leq \operatorname{deg}(A)-2$ (for integrability), consider the partial fraction decomposition $f=\sum_{k} b_{k} /\left(x-\lambda_{k}\right)$. Then, by linearity of $\mathcal{L}_{2}$,

$$
\mathcal{L}_{2}(f)=\mathcal{L}_{2}\left(\sum_{k} \frac{b_{k}}{x-\lambda_{k}}\right)=\sum_{k} \frac{b_{k}}{x-\frac{\lambda_{k}^{2}-1}{2 \lambda_{k}}} .
$$

Assume that the poles of $\mathcal{L}_{2}^{n}(f)$ remain simple. Then

$$
\mathcal{L}_{2}^{n} f \rightarrow \sum_{k} \frac{b_{k}}{x-\lambda_{\infty, k}}
$$

where $\lambda_{\infty, k}$ is either $i$ or $-i$ depending on the sign of $\operatorname{Im} \lambda_{k}$. Thus, for some constants $c_{ \pm}$,

$$
\mathcal{L}_{2}^{n} f \rightarrow \frac{c_{+}}{x-i}+\frac{c_{-}}{x+i}=\frac{c}{x^{2}+1}
$$


where the last equality follows from Theorem 2.7 and the fact that a Landen transformation preserves integrability.

The general proof requires some machinery from complex analysis. To this end, identify the integral of the rational function $f=B / A$ over the real line with the integral of the holomorphic 1-form $\phi=f(z) d z$ over the real projective line $\mathbb{R} P^{1}$; that is,

$$
\int_{-\infty}^{\infty} f(x) d x=\int_{\mathbb{R} P^{1}} \phi,
$$

where $\mathbb{R} P^{1}$ is the completed real axis sitting inside the Riemann sphere $\mathbb{C} P^{1}$. Recall that $f$ being a rational function corresponds to a holomorphic function on $\mathbb{C} P^{1}$.

The next required concept is that of a pull-back of a holomorphic 1-form. After the definition, recall the change of variables formula that connects the integral of a 1-form to that of its pull-back. The reader will find all these concepts in [10].

Definition 5.3. Let $\phi$ be a 1-form on a Riemann surface $S$, and $\pi: S \rightarrow T$ a holomorphic mapping between Riemann surfaces. The pull-back of $\phi$ induced by $\pi$ is defined as

$$
\left.\pi_{*} \phi\right|_{U}=\sum_{i=1}^{k} \sigma_{i}^{*} \phi
$$

on all $U \subset T$ simply connected and containing no critical values of $\pi$. Here $\sigma_{1}, \ldots, \sigma_{k}: U \rightarrow S$ are the distinct sections of $\pi$.

It is an elementary consequence of this definition that for holomorphic mappings $\pi_{1}: S_{2} \mapsto S_{3}$ and $\pi_{2}: S_{1} \mapsto S_{2}$,

$$
\pi_{1 *} \pi_{2 *} \phi=\left(\pi_{1} \circ \pi_{2}\right)_{*} \phi
$$

for any 1-form $\phi$ on $S_{1}$. The next lemma concerning holomorphic pull-backs and their path integrals is again an immediate consequence of the definition.

Lemma 5.3. If $\pi: S \rightarrow T$ is a holomorphism of Riemann surfaces, and $\phi$ is a holomorphic 1-form on $S$, then $\pi_{*} \phi$ is a holomorphic 1-form on T. Furthermore, for any oriented rectifiable curve $\gamma$ on $T$, the identity

$$
\int_{\gamma} \pi_{*} \phi=\int_{\pi^{-1} \gamma} \phi
$$

holds.

Let $f=B / A$ be a rational function. The pull-back of the 1 -form $\phi=f(z) d z$ on $\mathbb{C} P^{1}$ induced by $R_{m}: \mathbb{C} P^{1} \rightarrow \mathbb{C} P^{1}$ is the 1 -form

$$
\left(R_{m}\right)_{*} \phi=\sum_{j=1}^{m} \frac{B\left(\omega_{j}(y)\right)}{A\left(\omega_{j}(y)\right)} \omega_{j}^{\prime}(y) d y .
$$

Note that the right-hand side of (2.5) is precisely the integral of $\left(R_{m}\right)_{*} \phi$ over the projective real line. In this case, Lemma 5.3 amounts to $(2.5)$. The map $\mathcal{L}_{m}$ may therefore be identified with $\left(R_{m}\right)_{*}$. The following is a restatement of (5.1).

Theorem 5.4. Let $\phi$ be a holomorphic 1-form in a neighborhood $U$ of $\mathbb{R} P^{1} \subset \mathbb{C} P^{1}$. Then

$$
\lim _{n \rightarrow \infty}\left(R_{m}\right)_{*}^{n} \phi=\frac{1}{\pi}\left(\int_{\mathbb{R} P^{1}} \phi\right) \frac{d z}{z^{2}+1}
$$

where the convergence is of order $m$ and uniform on compact subsets of $U$. 
Remark 5.5. Let $f=B / A$ be a rational function such that its integral over the real line is finite. Then the 1 -form $f(z) d z$ on $\mathbb{C} P^{1}$ is holomorphic on some open set $U \supset \mathbb{R} P^{1}$. In particular, for $\beta=\min \{|\operatorname{Im} x|: A(x)=0\}$, and $N=\max \{|x|$ : $A(x)=0\}, f(z) d z$ is holomorphic on

$$
V_{\epsilon}=\left\{x \in \mathbb{C} P^{1}:|\operatorname{Im} x|<\beta-\epsilon\right\} \cup\left\{x \in \mathbb{C} P^{1}:|x|>N+\epsilon\right\}
$$

for all $\epsilon<\beta$.

A statement equivalent to Theorem 5.4 is proved now. This follows from conjugation by the map $M(z)=\frac{z+i}{z-i}$. Recall that $R_{m}=M^{-1} \circ f_{m} \circ M$, see for instance [7], where $f_{m}(z)=z^{m}$, so that $R_{m}^{n}=M^{-1} \circ f_{m}^{n} \circ M$ and therefore

$$
\lim _{n \rightarrow \infty}\left(R_{m *}\right)^{n} \phi=M_{*}^{-1} \lim _{n \rightarrow \infty}\left(f_{m *}\right)^{n} \phi_{1}
$$

where $\phi_{1}=M_{*} \phi$. On the other hand, one verifies that

$$
M_{*} \frac{d z}{z^{2}+1}=-\frac{d z}{2 i z} .
$$

Finally, observe that, by Lemma 5.3,

$$
\int_{\mathbb{R} P^{1}} \phi=\int_{\mathbb{R} P^{1}} M_{*}^{-1} \phi_{1}=-\int_{S^{1}} \phi_{1}
$$

where $S^{1}$ denotes the path that rotates once around the unit circle in counterclockwise direction. Theorem 5.4 is therefore equivalent to the following statement.

Theorem 5.6. Let $\phi$ be a holomorphic 1-form in a neighborhood $U$ of $S^{1}$. Then

$$
\lim _{n \rightarrow \infty}\left(f_{m}\right)_{*}^{n} \phi=\frac{1}{2 \pi i}\left(\int_{S^{1}} \phi\right) \frac{d z}{z}
$$

where the convergence is of order $m$ and uniform on compact subsets of $U$.

Proof. Using the local coordinate $z$, write $\phi=\phi(z) d z$ where $\phi(z)$ is analytic on an annulus $\frac{1}{R}<|z|<R$ for some $R>1$. The function $\phi(z)$ admits a Laurent expansion

$$
\phi(z)=\sum_{k=-\infty}^{\infty} a_{k} z^{k}
$$

and the coefficients $a_{k}$ satisfy

$$
\|\phi\|:=\sum_{k=-\infty}^{\infty}\left|a_{k}\right| R^{|k|}<\infty
$$

Since

$$
a_{-1}=\frac{1}{2 \pi i} \int_{S^{1}} \phi(z) d z
$$

it is required to show that

$$
\lim _{n \rightarrow \infty}\left(f_{m}\right)_{*}^{n} \phi=a_{-1} \frac{d z}{z} .
$$

In order to verify this, start with

$$
\left(f_{m}\right)_{*} \phi=\sum_{j=1}^{m} \sigma_{j}^{*}(\phi(z) d z)=\sum_{k=-\infty}^{\infty} \sum_{j=1}^{m} a_{k} \sigma_{j}^{*}\left(z^{k} d z\right)=\sum_{k=-\infty}^{\infty} a_{k}\left(f_{m}\right)_{*}\left(z^{k} d z\right)
$$


where $\sigma_{1}(w), \ldots, \sigma_{m}(w)$ are the $m$-th root sections of $w=f_{m}(z)=z^{m}$, defined by

$$
\sigma_{j}(w)=e^{2 \pi i j / m} \sigma_{0}(w)
$$

where $\sigma_{0}(w)=w^{1 / m}$ is the value of the complex $m$-th root whose argument is between 0 and $2 \pi / m$. A direct calculation yields

$$
\begin{aligned}
\left(f_{m}\right)_{*}\left(z^{k} d z\right) & =\sum_{j=1}^{m} \sigma_{j}^{*}\left(z^{k} d z\right) \\
& =\sum_{j=1}^{m} e^{2 \pi i j k / m} w^{k / m}\left(\frac{1}{m} e^{2 \pi i j / m} w^{1 / m-1}\right) d w \\
& =\frac{1}{m}\left(\sum_{j=1}^{m} e^{2 \pi i j(k+1) / m}\right) w^{(k+1-m) / m} d w \\
& = \begin{cases}z^{(k+1-m) / m} d z & \text { if } m \mid k+1 \\
0 & \text { if } m \mid k+1\end{cases}
\end{aligned}
$$

This establishes the formula

$$
\left(f_{m}\right)_{*} \phi=\sum_{k=-\infty}^{\infty} a_{m(k+1)-1} z^{k} d z .
$$

Consequently,

$$
\begin{aligned}
\left\|\left(f_{m}\right)_{*}^{n} \phi-a_{-1} \frac{d z}{z}\right\| & =\left\|\sum_{k=-\infty}^{\infty} a_{m^{n}(k+1)-1} z^{k} d z-a_{-1} \frac{d z}{z}\right\| \\
& =\sum_{k=-\infty, k \neq-1}^{\infty}\left|a_{m^{n}(k+1)-1}\right| R^{|k|} \\
& =\sum_{k=-\infty, k \neq-1}^{\infty}\left|a_{m^{n}(k+1)-1}\right| R^{\left|m^{n}(k+1)-1\right|} \frac{R^{|k|}}{R^{\left|m^{n}(k+1)-1\right|}} \\
& \leq \frac{R}{R^{m^{n}}} \sum_{k=-\infty, k \neq-1}^{\infty}\left|a_{m^{n}(k+1)-1}\right| R^{\left|m^{n}(k+1)-1\right|} \\
& \leq \frac{R}{R^{m^{n}}}\left\|\phi-a_{-1} \frac{d z}{z}\right\| .
\end{aligned}
$$

As $n \rightarrow \infty$, this quantity converges to zero to order $m$.

Corollary 5.4. For a rational function $f$

$$
\int_{-\infty}^{\infty} f(x) d x=\pi \lim _{n \rightarrow \infty} \mathcal{L}_{m}^{n}(f)(0)
$$

provided that the integral is finite. In that case, convergence is of order $m$.

\section{ImPlementation}

In this section the implementation of the numerical scheme proposed in the previous sections is discussed. Assume that $A$ and $B$ are polynomials and let

$$
I:=\int_{-\infty}^{\infty} \frac{B(x)}{A(x)} d x
$$


The first issue under consideration is that the exact evaluation of the iterates of $\mathcal{L}_{m, p}^{n}(B / A)$ usually leads to extreme growth in the size of the coefficients. Therefore, while computing these iterates numerically, the coefficients are normalized after each iteration by keeping the denominator polynomial with leading coefficient 1.

Example 6.1. This continues Example 2.6 where

$$
\int_{-\infty}^{\infty} \frac{d x}{x^{2}+4 x+15}=\frac{\pi}{\sqrt{11}}
$$

is approximated using $\mathcal{L}_{2,2}$. The first iterate is $\frac{32}{60 x^{2}+112 x+240}$. The next two Landen iterates are

$$
\frac{19200}{57600 x^{2}+40320 x+77456}, \quad \frac{5186150400}{17845862400 x^{2}+1601187840 x+16614420736} .
$$

Experiments show that the number of digits in the coefficients grows exponentially even if common factors are cancelled.

Fix $m \in \mathbb{N}$ and let $p=\operatorname{deg}(A)$. The normalization proceeds as follows: let

$$
b_{0}=\left(b_{0,0}, b_{0,1}, \ldots, b_{0, p-2}\right) \text { and } a_{0}=\left(a_{0,0}, a_{0,1}, \ldots, a_{0, p}\right)
$$

be the coefficients of the initial rational function $B / A$. For $n \geq 1$, let

$$
\left(b_{n}, a_{n}\right)=\frac{1}{a_{n, 0}^{\prime}}\left(b_{n}^{\prime}, a_{n}^{\prime}\right), \quad \text { where }\left(b_{n}^{\prime}, a_{n}^{\prime}\right)=\mathcal{L}_{m, p}\left(b_{n-1}, a_{n-1}\right) .
$$

Recall that the rational Landen transformations preserve the degree of the denominator. Hence, $a_{n, 0}^{\prime} \neq 0$ throughout. If the integral of $B / A$ converges, then by Corollary 5.4 its value is given by $\pi \lim _{n \rightarrow \infty} b_{n, 0}$.

Proposition 5.1 shows that, if the integral over $B / A$ is finite, then $a_{n}$ converges to the coefficients $c_{k}$ of one of the $p+1$ candidates

$$
(x-i)^{k}(x+i)^{p-k}, \quad 0 \leq k \leq p,
$$

depending on the number $k$ of roots of $A$ with positive imaginary part. In particular, Corollary 5.2 shows that if all coefficients are real, then $a_{n}$ will converge to

$$
\left(\left(\begin{array}{c}
p / 2 \\
0
\end{array}\right), 0,\left(\begin{array}{c}
p / 2 \\
1
\end{array}\right), 0, \ldots,\left(\begin{array}{c}
p / 2 \\
p / 2-1
\end{array}\right), 0,\left(\begin{array}{c}
p / 2 \\
p / 2
\end{array}\right)\right) .
$$

Conversely, if convergence to one of the candidates in (6.4) is observed, then the invariance of the integral under $\mathcal{L}_{m}$ shows that the initial integral must be finite. Thus the algorithm also detects the integrability of rational functions.

After each step, the implementation checks if the approximate relative error $\left|\left(b_{n, 0}-b_{n-1,0}\right) / b_{n, 0}\right|$ is less than the precision goal. The distance of the coefficients $a_{n}$ to their limiting values is also monitored. While computations may be made symbolically (provided that the initial coefficients are known exactly) it is generally sensible to work with a fixed precision throughout. For most integrands (compare Examples 2.6 and 4.1) this working precision need only be slightly larger, say 5 digits, than the precision goal. In case of almost singular integrands (compare Example 4.2) the working precision may need to be chosen somewhat higher. For the problems considered by the authors, a reasonable choice (currently used as a default in the Mathematica implementation mentioned below) seems to be 20 additional digits. The implementation of the Landen iteration method is given below in pseudo code. 
Input: the coefficients $\left(b_{0}, a_{0}\right)$ of the rational function $B / A$ of degree $p$, the order $m$ of the method, and the precision goal $\epsilon>0$.

Output: an approximation (produced using Landen iterations of order $m$ ) to the integral of $B / A$ over the real line with (approximate) relative error less than $\epsilon$.

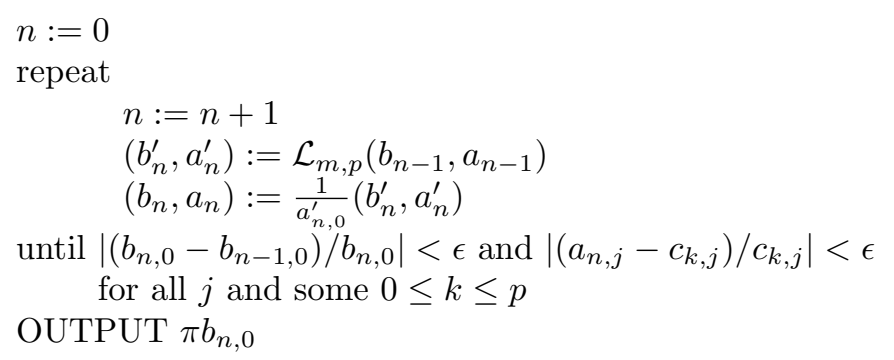

The described method for integrating rational functions over the real line has been implemented in Mathematica and can be downloaded from the website

http://www. math.tulane.edu/ vhm

The Landen transformations can be either generated by the package, following the description in Section 2, or downloaded as well. The following examples demonstrate the basic usage of this package. Further examples can be obtained from the above website.

Example 6.2. Given a rational function $f$, its integral over the entire real line can be computed using the function NLandenIntegrate. For instance, to compute the integral of $1 /\left(x^{2}+4 x+15\right)$ to a precision of 100 digits, input:

NLandenIntegrate [1/( $\left.x^{\wedge} 2+4 x+15\right)$, PrecisionGoal->100]

$>0.94722582509948293642963438181697406661998807 \ldots$

By default the Landen transformation is chosen to be of order 2 (see Remark 3.3 for why this is desirable) but higher orders $m$ may be used by setting MethodOrder->m. Further options exist to control the number of iterations, set the working precision manually, or to obtain the intermediate Landen iterates.

Example 6.3. Before using the function NLandenIntegrate demonstrated in the previous example the corresponding Landen transformation needs to be available. The command

GenerateLandenTransforms [10]

will generate the Landen transformations of order 2 for degrees up to 10 . Note that on a modern desktop computer this will take less than half a second. After execution, the Landen transformations are directly available as follows, compare example 2.1:

LandenStep $[\{\{\mathrm{b} 0\},\{\mathrm{a} 0, \mathrm{a} 1, \mathrm{a} 2\}\}, 2]$

$>\left\{\{2 \mathrm{a} 0 \mathrm{~b} 0+2 \mathrm{a} 2 \mathrm{~b} 0\},\left\{4 \mathrm{a} 0 \mathrm{a} 2,-2 \mathrm{a} 0 \mathrm{a} 1+2 \mathrm{a} 1 \mathrm{a} 2, \mathrm{a}^{\wedge} 2-\mathrm{a} 11^{\wedge} 2+2 \mathrm{a} 0 \mathrm{a} 2+\mathrm{a} 2 \wedge 2\right\}\right\}$

Again, higher orders than 2 can be generated using the option MethodOrder. Once generated, these Landen transformations may be stored to a file. Alternatively, pregenerated Landen transformations are available for download.

\section{Conclusions}

A numerical method for the integration of rational functions on the real line has been described. The method has order of convergence prescribed by the user. 
Its convergence and robustness have been analyzed. Examples illustrating speed of convergence as well as the flexibility of this method have been provided. A Mathematica package is available for the general public.

Future work will attempt to couple this method with Pade approximations of the integrand to produce a highly efficient numerical scheme for smooth integrable functions. The construction of a numerical scheme for the finite interval case requires the theory of Landen transformations on a half-line. This is an open question.

Acknowledgments. The first author is partially funded by the AARMS Director's Postdoctoral Fellowship. The work of the third author was partially funded by NSF-DMS 0070567 and the second and last authors were funded, as graduate students, by the same grant.

\section{REFERENCES}

[1] E. Beyerstedt, N. Cayco-Gajic, N. Kallus, L. Medina, V. Moll, and J. Stigile. Numerical integration with rational Landen transformations. In preparation, 2009.

[2] J. M. Borwein and P. B. Borwein. Pi and the AGM-A study in Analytic Number Theory and Computational Complexity. Wiley, New York, 1st edition, 1987.

[3] L. Castro, C. Enrique, G. Koffi, C. Price, L. Medina, and V. Moll. Landen transformation with cubic convergence. In preparation, 2009.

[4] M. Chamberland and V. Moll. Dynamics of the degree six Landen transformation. Discrete and Dynamical Systems, 15:905-919, 2006.

[5] R. Garcia, A. Nguyen, I. Ojeda, B. Wilson, C. Price, L. Medina, and V. Moll. Landen transformation with quartic convergence. In preparation, 2009.

[6] K. F. Gauss. Arithmetisch Geometrisches Mittel. Werke, 3:361-432, 1799.

[7] D. Manna and V. Moll. Rational Landen transformations on $\mathbb{R}$. Math. Comp., 76:2023-2043, 2007.

[8] D. Manna and V. Moll. Landen Survey. MSRI Publications: Probabilty, Geometry and Integrable Systems. In honor of Henry McKean 75th birthday, 55:201-233, 2008.

[9] V. Moll. The evaluation of integrals: a personal story. Notices of the AMS, 49:311-317, 2002.

[10] I. Shafarevic. Basic Algebraic Geometry. Varieties in Projective Space. Springer Verlag, New York, second edition, 1994.

Department of Mathematics and Computer Science, Virginia Wesleyan College, NorFOLK, VA 23502

E-mail address: dmanna@vwc.edu

Department of Mathematics, Rutgers University, New Brunswick, NJ 08854-8019

E-mail address: lmedina@math.rutgers.edu

Department of Mathematics, Tulane University, New Orleans, LA 70118

E-mail address: vhm@math.tulane.edu

Department of Mathematics, Tulane University, New Orleans, LA 70118

E-mail address: astraub@math.tulane.edu 